\title{
Uncertainty quantification and validation of 3D lattice scaffolds for computer-aided biomedical applications
}

\author{
Recep M. Gorguluarslan, Seung-Kyum Choi*, Christopher J. Saldana \\ The G. W. Woodruff School of Mechanical Engineering, Georgia Institute of Technology, Atlanta, GA 30332, USA
}

\section{A R T I C L E I N F O}

\section{Keywords:}

Bone scaffold

Lattice structure

Additive manufacturing

Uncertainty quantification

Validation

\begin{abstract}
A B S T R A C T
A methodology is proposed for uncertainty quantification and validation to accurately predict the mechanical response of lattice structures used in the design of scaffolds. Effective structural properties of the scaffolds are characterized using a developed multi-level stochastic upscaling process that propagates the quantified uncertainties at strut level to the lattice structure level. To obtain realistic simulation models for the stochastic upscaling process and minimize the experimental cost, high-resolution finite element models of individual struts were reconstructed from the micro-CT scan images of lattice structures which are fabricated by selective laser melting. The upscaling method facilitates the process of determining homogenized strut properties to reduce the computational cost of the detailed simulation model for the scaffold. Bayesian Information Criterion is utilized to quantify the uncertainties with parametric distributions based on the statistical data obtained from the reconstructed strut models. A systematic validation approach that can minimize the experimental cost is also developed to assess the predictive capability of the stochastic upscaling method used at the strut level and lattice structure level. In comparison with physical compression test results, the proposed methodology of linking the uncertainty quantification with the multi-level stochastic upscaling method enabled an accurate prediction of the elastic behavior of the lattice structure with minimal experimental cost by accounting for the uncertainties induced by the additive manufacturing process.
\end{abstract}

\section{Introduction}

Current standard orthopedic implants are usually made of fully dense metal alloys, which are much stiffer than the actual bone. This stiffness mismatch causes issues such as stress-shielding due to the increased stresses on the surrounding bone tissue, thus resulting in bone resorption and loosening of implants (Kowalczyk, 2001). The stiffness or modulus of a metal implant material can be reduced by introducing cellular lattice structures, which possess lightweight and high strength material characteristics due to their porous network with interconnected strut members (Deshpande et al., 2001; Gorguluarslan et al., 2016; Hammetter et al., 2013). Metal orthopedic implants designed with the periodic lattice cells have recently gained interest in the biomedical community since their repeating unit-cell structure can be tailored to have similar mechanical characteristics as that of bone (Arabnejad and Pasini 2012). The interconnected pore structure of the lattice structures can also promote bone tissue ingrowth for long term fixation of orthopedic bone implants (Li et al., 2006). Recently, porous metal scaffolds have gained importance, especially for load-bearing orthopedic implant applications to enable the fixation of the implants established with the ingrowth of bone tissues (Heinl et al., 2008; Parthasarathy et al., 2010). With the recent advancements in additive manufacturing (AM) technologies, it is now possible to fabricate the metal scaffolds designed with lattice cells that possess high geometric complexity. To fabricate lattice scaffolds made of biocompatible metals such as titanium alloys, two AM techniques gained popularity, namely Electron Beam Melting (EBM) (Heinl et al., 2008; Parthasarathy et al., 2010) and Selective Laser Melting (SLM) (Arabnejad et al., 2016; Challis et al., 2010; Mullen et al., 2010; Wieding et al., 2014). Tissue ingrowth due to the porous structure of lattice scaffolds also paves the way for the restoration of various anatomic defects in bone tissue engineering using some biodegradable artificial materials, such as polycaprolactone (PCL) blends fabricated by Selective Laser Sintering (Doyle et al., 2015, 2014; Eshraghi and Das, 2012) and Fused Deposition Modeling (FDM) (Senatov et al., 2016; Zein et al., 2002). Thus, AM-fabricated porous scaffolds gain great importance for tissue engineering as well as for implants to enable tissue ingrowth in biomedical applications (Giannitelli et al., 2014; Hollister, 2009).

The ease of fabrication using AM technologies has recently stimulated efforts to model and characterize the basic properties of such

\footnotetext{
* Corresponding author.

E-mail addresses: rmg@gatech.edu (R.M. Gorguluarslan), schoi@me.gatech.edu (S.-K. Choi), christopher.saldana@me.gatech.edu (C.J. Saldana).
} 
scaffolds designed with lattice structures that will be fabricated by AM. Existing studies show that there exist geometrical irregularities and imperfections in the fabricated lattice structures, which result in geometrical differences between the finite element (FE) model of the structure and the fabricated one when AM techniques are used in the fabrication (Cahill et al., 2009; Cansizoglu et al., 2008; Harrysson et al., 2008). Cansizoglu et al. (2008) reported the effect of the build angle and orientation on overall material properties in lattice materials fabricated using the Electron Beam Melting (EBM) process. Harrysson et al. (2008) showed how the members (i.e. struts) of lattice structures in AM-fabricated implants have a rough texture with varying crosssections, which result in inaccurate FE model predictions if not considered. Cahill et al. (2009) found that the FE models of scaffolds with a rough surface on the struts resulted in better predictions than the models with smooth strut surfaces when compared to experimental results. Similarly, Campoli et al. (2013) and Karamooz Ravari et al. (2014) showed that the FE model simulations with the geometric variations in the strut diameter along the strut length agreed well with the experimental results. Thus, it is apparent that the imperfections and variations on the strut geometry must be taken into account in the FE modeling and design process.

Despite that the characterization of the lattice structure performance with variations at strut level produced good predictions, these existing works were carried out in a deterministic setting. Uncertainty, however, gains importance in lattice scaffolds because there is a higher probability of introducing manufacturing error when lattice structures are fabricated by the AM processes. Since the length scale of the fabricated strut members of a lattice structure is usually very small, the mechanical responses of the fabricated structures will be much more sensitive to the unavoidable uncertainties. For this reason, it is necessary to account for the uncertainties when predicting the mechanical response of scaffolds designed by lattice structures. Recent years have also witnessed a growing research interest in the study of lattice structure modeling considering uncertainties. Park et al. (2014) accounted for the variations of the diameters at each fabricated layer in the FDM process to generate an FE model of the individual struts using voxel elements and predicted the equivalent strut diameters based on the simulation results of these models. The deterministic homogenized diameter values of the individual struts are passed onto the unit lattice cell model to predict the effective material properties of the lattice structures. Gorguluarslan et al. (2015) presented a multi-level stochastic upscaling method to capture the effects of strut level geometric uncertainties on the lattice level material characterization of lattice structures fabricated by FDM. These studies, however, consider only the effect of uncertainties on the strut geometry (i.e. to find the homogenized strut diameter) based on the experience of the user. For more accurate predictions from the models, the uncertainties, induced by the AM process, must be quantified based on the fabricated parts for better accuracy from the FE models. Moreover, the uncertainties must be considered not only for variables that influence the geometric proper- ties but also for the ones that affect the material properties of individual struts such as porosity. A systematic validation approach that can enable the prediction accuracy assessment at multiple scale levels under uncertainties is also required for minimizing the experimental cost.

The current work is the first attempt along this direction for scaffolds designed by lattice structures. The primary goal of this study is to develop an uncertainty quantification approach to accurately model the realistic mechanical behavior of lattice scaffolds that must support mechanical loading in biomedical applications by accounting for the strut level uncertainties introduced by the AM process. Computer simulations and experimental measurements, which are two interrelated approaches, are used to gain knowledge on the uncertainties in the strut level geometry and material porosity and on their correlation to homogenized properties of struts and lattice structures. Specifically, the uncertainties are quantified from the high-resolution strut models reconstructed from computer tomography (CT)-scanned images of fabricated lattice structures that can capture the real strut geometry and microstructure. A multi-level stochastic upscaling method (Gorguluarslan et al., 2015) is utilized to propagate the uncertainties from strut level to the lattice level to efficiently predict the macroscale properties of the lattice structures. A systematic validation approach that can minimize the experimental cost is also utilized to quantitatively evaluate the prediction capability of the computational models used in the multi-level upscaling process of the lattice structures. Accurate prediction of the uncertainties on the overall elastic modulus of the lattice structure, which has a considerable importance to design lattice scaffolds, is considered as the primary focus of the presented approach. The proposed methodology can be generalized to apply any material and AM technique that can be used to develop lattice scaffolds which will incorporate computationally intensive simulations and expensive experiments associated with the uncertainties.

\section{Materials and methods}

\subsection{Test specimens}

The scaffold specimen analyzed in this study is comprised of the body-centered cubic (BCC) lattice cells given in Fig. 1(a). The strut diameter $D$ and length $L$ are designed to be $0.5 \mathrm{~mm}$ and $4 \mathrm{~mm}$, respectively, for each BCC unit cell. The complete lattice structure has $n=3$ BCC cells in each direction, which gives overall dimensions of $12 \times 12 \times 12 \mathrm{~mm}^{3}$. The corresponding lattice structure model, filled by $3 \times 3 \times 3$ BCC unit cells, is modeled in the commercial finite element (FE) modeling software ABAQUS as shown in Fig. 1(b). Selective Laser Melting (SLM) $280 \mathrm{HL}$, which has a maximum laser output power of $1000 \mathrm{~W}$ and a scanning speed of $15 \mathrm{~m} / \mathrm{s}$, has been used to fabricate the metal lattice structures as shown in Fig. 1(c). In order to identify the elastic properties of the specimens fabricated by SLM, a standard quasistatic uniaxial compression test was conducted. The force $\left(F_{\text {exp }}\right)$ and

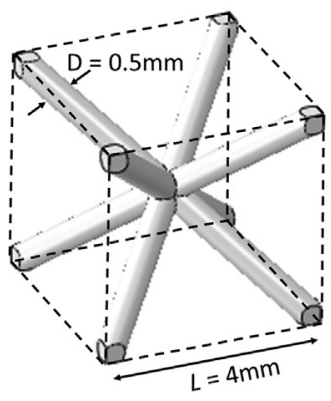

(a)

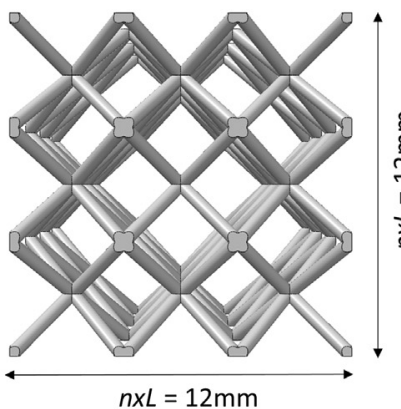

(b)

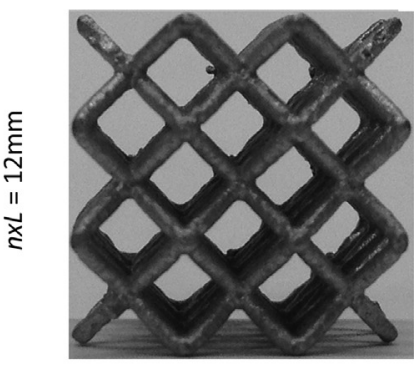

(c)

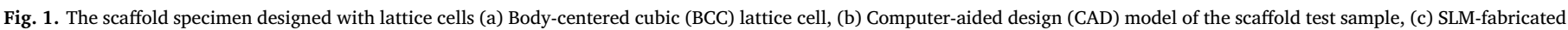
scaffold. 


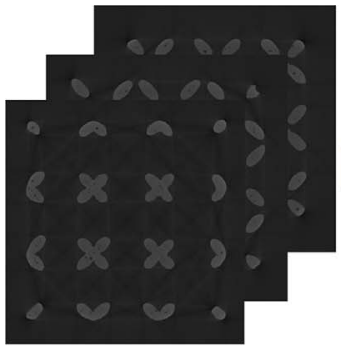

(a) 2D scanned images

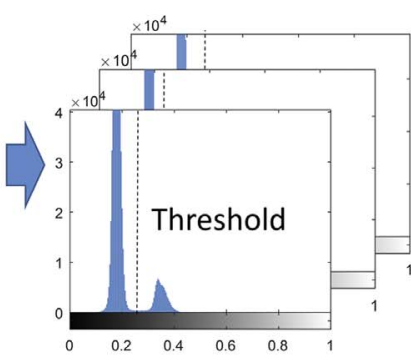

(b) Gray level

histograms

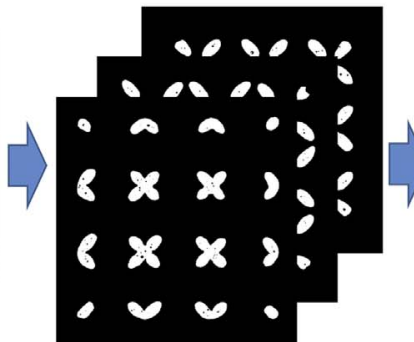

(c) Segmentation process

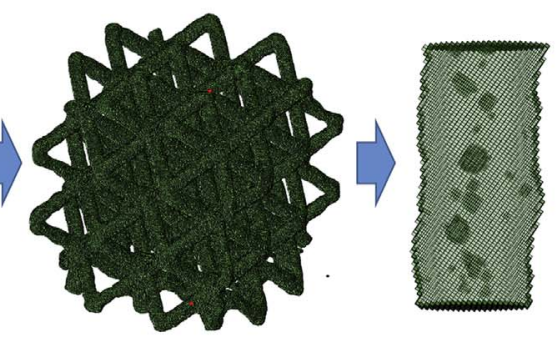

(e) Extracted

(d) 3D Voxel Model Construction Strut models

Fig. 2. Schematic diagram of the 3D model reconstruction process using the 2D scanned image data.

change in gauge length (i.e. compressive strain) were recorded as the experimental results. Uniaxial compression engineering stress was calculated as the ratio of the force to one surface area with $A_{\text {lattice }}=144 \mathrm{~mm}^{3}$. Then, the effective elastic modulus $\left(E_{\text {lattice }}\right)$ was calculated through the compression tests by

$E_{\text {lattice }}=\frac{\sigma}{\varepsilon}=\frac{\sigma_{b}-\sigma_{a}}{\varepsilon_{b}-\varepsilon_{a}}$

where $\varepsilon$ is the compression engineering strain, $\sigma_{a}$ is the stress at $\varepsilon_{a}$ and $\sigma_{b}$ is the stress at $\varepsilon_{b}$.

\subsection{CT scanning and $3 D$ model reconstruction}

High-resolution scans of the fabricated scaffolds were conducted using a computer tomography (CT) scanner (Zeiss metrotom 800, Georgia Institute of Technology, USA) at a resolution of approximately $13.53 \mu \mathrm{m}$. The 3D models of the fabricated scaffolds and individual struts are reconstructed from the 2D scan images using a reconstruction process depicted in Fig. 2. The first step of this reconstruction process is to collect the 2D scanned images (Fig. 2(a)). The solid and void phase can be distinguished by thresholding based on the gray level histograms of the images as shown in Fig. 2(b). A widely used automated segmentation algorithm, the Otsu method (Otsu, 1979), is implemented for this purpose. The segmented binary images, generated based on the calculated threshold values (Fig. 2(c)), are then combined and converted to a high-resolution $\mathrm{FE}$ mesh with 8-noded hexahedral voxel elements, as shown in Fig. 2(d), using an in-house developed algorithm that converts the binary image information to the voxel model. Then, the individual strut models, as shown in Fig. 2(e), are extracted to quantify the uncertainties. To construct the 3D model of lattice structures, the CT-scan images were manipulated to have an element length of $27.06 \mu \mathrm{m}$ to reduce the computational cost of the reconstruction process. Measurements are obtained from the individual strut models for morphological analysis to characterize the uncertainties of the surface irregularities (or roughness) as well as the material porosity. The strut level uncertainties are investigated in terms of three main variables in this study as explained in Section 3.1: (1) strut diameter uncertainty, (2) strut angle uncertainty that gives the deviation from the center of each fabricated layer, and (3) porosity of the strut material. The combination of the uncertainties of the first two variables will result in the surface roughness in the voxel-based strut model. The uncertainty of porosity in fabricated struts is investigated in terms of pore volume and pore size (i.e. the equivalent spherical diameter, defined as the diameter of a sphere of the same volume).

\subsection{Uncertainty quantification}

The term Uncertainty Quantification (UQ) could encompass the whole field of statistics since it defines the process of identification, quantitative characterization, and reduction of uncertainties in compu- tational and real world applications (Oberkampf and Roy, 2010). The UQ can be viewed as two different types of coupled components. The first is the inverse assessment of the input uncertainty, where the statistical parameters are estimated based on available data. The second is the forward propagation of uncertainty from computational model input parameters to model outputs (Najm, 2009). The focus of this study is on both the uncertainty quantification of input parameters and the propagation to model outputs, as well as across multiple scale levels.

\subsubsection{Bayesian information criterion}

In cases where experimental measurements or statistical data are available for a random input variable, uncertainty quantification is accomplished through statistical modeling, where the uncertainty is expressed as a probability distribution with some statistical parameters (Choi et al., 2006). Model selection methods have been developed for this purpose, in which the optimal distribution model, $D$, and the corresponding statistical parameters, $\hat{\theta}$, are determined among $N_{D}$ number of distribution models for a random variable $X$ with the available statistical data $X=\left\{x_{1}, x_{2}, \ldots, x_{n}\right\}$ based on Maximum Likelihood Estimation (MLE) (Myung, 2003). In particular, the logarithm of the likelihood value, $\ln L$, is calculated for each distribution model as the information loss $I L_{M L E}\left(D_{j} \mid X\right)=\ln L=\ln p\left(X \mid \hat{\theta}_{j}, D_{j}\right)$ over $j$ where $j=1,2, \ldots, N_{D}$ to choose the most plausible distribution model for the data. The use of MLE in model selection favors the distribution models with more statistical parameters, making it inefficient for selecting the best-fitted distribution (Beck and Yuen, 2004). Bayesian Information Criterion (BIC) method (Schwarz, 1978) has a higher convergence to the true model since it also considers the number of samples, denoted by $n$, as well as the number of statistical parameters, denoted by $k_{p}$, in the calculation of the information loss:

$I L_{B I C}\left(D_{j} \mid X\right)=-2 \ln p\left(X \mid \hat{\theta}_{j}, D_{j}\right)+k_{p, j} \ln n$

Once various distribution models are fitted to the data and the corresponding BIC values are calculated, the distribution type with the smallest BIC value is chosen as the best fit for the data of the input variable.

\subsection{Stochastic upscaling method}

The quantification of uncertainties can be achieved inversely where the input uncertainties are determined using the known uncertainties of model responses in multiscale modeling with a stochastic upscaling method (Gorguluarslan and Choi, 2014). The basic idea of the upscaling procedure is as follows. A coarse scale model $Y_{C M}(\widetilde{\omega})=f_{C M}\left(X_{C M}(\eta)\right)$ that produces the stochastic output $Y_{C M}(\widetilde{\omega})$ with some stochastic homogenized input variables $X_{C M}(\eta)$ is identified to represent a fine scale model $Y_{F M}(\omega)=f_{F M}\left(X_{F M}(\theta)\right)$ in a lower dimension where $Y_{F M}(\omega)$ and $X_{F M}(\theta)$ denote the stochastic response and input variables, respectively. In 
these notations, $\theta, \omega, \eta$ and $\widetilde{\omega}$ denote the random quantities in the input and output of the fine scale and coarse scale models, respectively. In order to find the equality between the fine scale and the coarse scale models, the random input variables, $X_{C M}(\eta)$, can be identified such that $Y_{F M}\left(X_{F M}(\theta)\right)=Y_{C M}\left(X_{C M}(\eta)\right)$. Then, an optimization procedure can be applied to find the homogenized parameters, $X_{C M}$. When we introduce the exponential of the squared distortion as an objective function, the corresponding optimization statement can be written as

$X_{C M}(\hat{\eta})=\arg \max _{X_{C M}} e^{-\left(Y_{C M}-Y_{F M}\right)^{2}}$

subject to $\left|M_{C M}^{(t)}-M_{F M}^{(t)}\right| \leq \varepsilon_{c}$

where $\hat{\eta}$ denotes the estimated randomness in the coarse scale model when the optimization is used and $M_{C M}^{(t)}$ and $M_{F M}^{(t)}$ denote the $t$ th statistic moments of the responses at coarse and fine scale, respectively. $\varepsilon_{c}$ is the user-defined error criterion for the desired accuracy. Polynomial Chaos Expansion (PCE) (Choi et al., 2006) can be utilized to reduce the dimensionality in the optimization, which increases due to the uncertainty of the input variables. Then, the coarse scale random variable is written as

$X_{C M}(\hat{\eta})=\sum_{k=0}^{P} b_{k} \Phi_{k}(\xi(\hat{\eta}))$

where $b_{k}$ are undetermined coefficients and $\Phi_{k}$ denote orthogonal polynomials. Depending upon the type of random variables, $\xi$, the orthogonal polynomials, can be selected. If the sampling methods are utilized to generate data for $n$ number of samples (e.g. Latin Hypercube Sampling (LHS) (Mckay et al., 2000)), then the coarse scale output samples are denoted by $Y_{C M}=\left\{y_{C M}(1), y_{C M}(2), \ldots, y_{C M}(n)\right\}$ and fine scale output samples are $Y_{F M}=\left\{y_{F M}(1), y_{F M}(2), \ldots, y_{F M}(n)\right\}$. When the coarse scale random variable, $X_{C M}$, is projected onto the PCE, the objective function can be rewritten as

$\hat{U}(b)=\frac{1}{n} \sum_{j=1}^{n} \exp \left\{-\left(f_{C M}\left(\sum_{k=0}^{P} b_{k} \Phi_{k}(\xi(j))\right)-y_{F M}(j)\right)^{2}\right\}$

where $j=1,2, ., n$. Thus, the PCE coefficients, $b_{k},(k=0,1, \ldots, P)$, are the variables that will be determined in the optimization process. This optimization procedure implies that the objective function in Eq. (5) is at its maximum when all the coarse scale output values are equivalent to the corresponding fine scale output values. To achieve this, the design variables, $b_{k}$, are changed in the optimization process in a way that the coarse scale model can accurately approximate the fine scale outputs (Gorguluarslan et al., 2015). This optimization process is similar to coarse-graining approaches where information theory is utilized to find the optimal coarse-grained model parameters with the least information loss by using, for instance, relative entropy (Shell, 2008). A validation approach is necessary to be able to quantitatively evaluate the predictions made by this upscaling approach with minimal experiment cost under uncertainties. For this purpose, we presented a multi-level validation and upscaling framework in Section 2.6 by incorporating the validation step introduced in Section 2.5 with the upscaling method.

\subsection{Validation under uncertainties}

Validation is known as the assessment of the prediction accuracy of computational models through comparison with physical experiments. One key fundamental challenge in validation efforts is to compare the model predictions with corresponding experimental observations given there being uncertainties in both. An appropriate validation metric is required to make this comparison quantitatively. Various types of quantitative metrics have been proposed over the years for the validation of computational models (Oberkampf and Roy, 2010). Among those metrics, the $u$-pooling method (Ferson et al., 2008) has gained interest as it can be applicable to the case of multiple validation sites. In a validation problem with multiple validation sites, the experimental observations are denoted by $Y_{E}^{(k)}=\left\{y_{E}^{(k)}(j)\right\}$ where $j=1, \ldots, n_{E}^{(k)}$ and $n_{E}^{(k)}$ is the number of experimental observations at $k^{\text {th }}$ validation site. Here, $k=1, \ldots, n_{V S}$ denotes the validation sites and $n_{V S}$ is the number of validation sites. For each experimental observation $y_{E}^{(k)}(j)$, a corresponding $u$-value is calculated using the corresponding prediction CDF model as

$u_{t}=F_{C M, i}^{(k)}\left(y_{E, i}^{(k)}(j)\right), t=1, \ldots, n_{T}$

where $n_{T}$ denotes the total number of experimental observations at all validation sites calculated by $n_{T}=\sum_{k=1}^{n_{V S}} n_{E}^{(k)}$. The corresponding CDF model of the predictions can be determined by using the BIC method described in Section 2.3.1 According to the probability integral transformation, if the model CDF accurately predicts the experimental observations, then the empirical CDF (ECDF) of the $u$-values, $F_{E}(u)$, must follow a standard uniform distribution (SUD).

The ECDF of the pooled $u$-values is compared with the CDF of the SUD to make a decision for validating predictions. The KolmogorovSmirnov (K-S) hypothesis testing method (Marsaglia et al., 2003) is proposed in this study to compare these two CDFs. A null hypothesis, $H_{0}$, is established in the K-S test by stating that the theoretical CDF of a specific distribution type accurately represents the ECDF of a given set of statistical data. The K-S test checks the null hypothesis by evaluating the maximum distance between the ECDF of the $u$-values, $F_{E}(u)$, and the CDF of SUD, denoted by $F_{S U D}(\mathrm{u})$ :

$d_{K S}=\max _{u}\left|F_{S U D}(u)-F_{E}(u)\right|$

Since $d_{K S}$ is a random variable, the CDF of $d_{K S}$ is related to a significance level $\alpha$ as

$P\left(d_{K S} \leq d_{K S}^{\alpha}\right)=1-\alpha$

for the confidence level, $1-\alpha$. Here, $d_{K S}^{\alpha}$ is a critical value obtained from standard mathematical tables given for K-S tests. The probability that $d_{K S}$ calculated from samples is smaller than $d_{K S}^{\alpha}$ is defined as the $p$-value, which can be used to test the null hypothesis. In the K-S test, since $d_{K S}$ is known to follow the Kolmogorov distribution, the $p$-value can be calculated from the Kolmogorov CDF at $d_{K S}$ (Marsaglia et al., 2003). If the calculated $p$-value is larger than $\alpha$, then the null hypothesis is $H_{O}=0$. This means the $u$ values are found to be drawn from the CDF of the SUD $\left(F_{S U D}(u)\right)$ (i.e. the model is accepted); otherwise, $H_{O}=1$ (i.e. the model is rejected) at a significance level of $\alpha$. The test accepts the null hypothesis more strongly when the $p$-value is larger. Since the K-S test statistic is calculated based on the number of experiments, it will provide an unbiased decision criterion in the validation process even if a small number of experiments is available.

\subsection{Multi-level upscaling and validation framework}

In a hierarchical multi-level modeling process, a top-down decomposition of the system or product at a macroscale level into multiple sub-levels (e.g. mesoscale, microscale, etc.) requires a hierarchical uncertainty quantification and validation approach to be developed. The proposed multi-level upscaling and validation framework is illustrated in Fig. 3 as a validation pyramid (Babuška et al., 2007) for the application to the scaffold structure discussed in Section 2.1. In Fig. 3, $i=1, \ldots, N$ denotes the scale level of interest where $N$ is the smallest scale level. The lattice structure model is decomposed into two sub-levels, the mesoscale level (i.e. strut level, $i=2$ ) and macroscale level (i.e. lattice level, $i=1$ ), to simplify the computational models. Thus, for the lattice structure application, the number of levels is $N=2$. The upscaling process described in Section 2.4 is used at multiple scale levels to pass the information at the finer scales to the macroscale level from bottom up. The upscaling step at each scale level follows a validation step to quantitatively evaluate the prediction accuracy. 


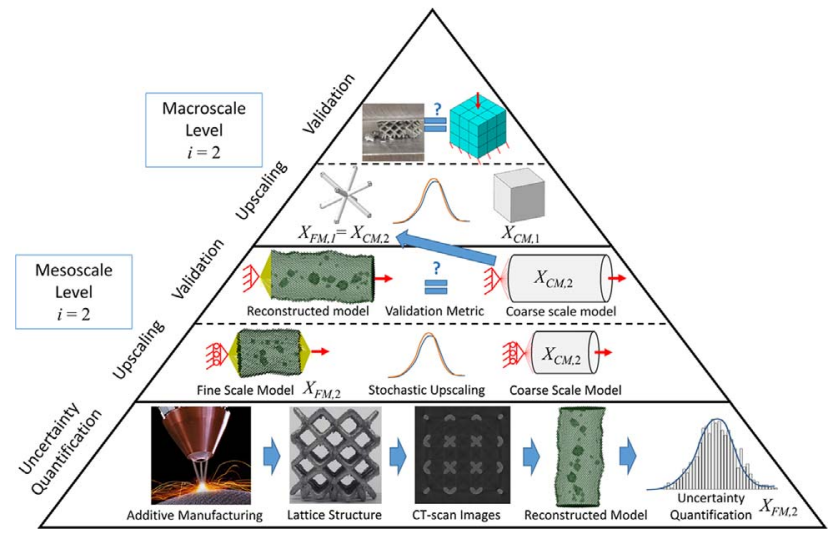

Fig. 3. Multi-level uncertainty quantification and validation approach in a validation pyramid.

At one scale level of interest, before the upscaling method is implemented, the uncertainties of the input variables of the fine scale model denoted by $X_{F M, i}$ must be quantified. At the smallest scale level, i.e. $i=N$, the uncertain data of the input variables are collected from the strut models obtained by the reconstruction process using the CT-scan images described in Section 2.2, as shown at the bottom level of the validation pyramid in Fig. 3. Then, the BIC method given in Section 2.3.1 is used to identify the stochastic parameters and the type of distribution that gives the best fit for the uncertain data of the fine scale input variables.

At the mesoscale level upscaling step $(i=2)$, first, the strut fine scale model is generated using the quantified uncertainties of the collected data and the FE simulations are conducted on this fine scale strut model. Then, the upscaling method described in Section 2.4 is implemented at strut level to predict the homogenized coarse scale model input variables denoted by $X_{C M, 2}$. For the BCC lattice specimen, the homogenized diameter and the elastic modulus are considered as the strut level homogenized variables. The statistical data of the homogenized coarse scale input variables, $X_{C M, i}$, are found by using the optimal PCE coefficients in Eq. (4), and propagated to the next level of interest as $X_{C M, i}=X_{F M, i-1}$.

Two consecutive upscaling processes are conducted to propagate the uncertainties on to the homogenized diameter and elastic modulus of the struts as shown in Fig. 4. At a fine scale, an FE modeling technique that fills each deposited layer of the fabricated strut with voxel elements (i.e. 8-node brick elements) is used to accurately model the geometric and material uncertainties in the strut, mimicking the SLM fabrication process. The strut level input uncertainties with the corresponding distributions and statistical parameters determined using BIC method are used to generate the fine scale models for Strut Model 1 and Strut Model 2 in Fig. 4. The length of the strut that will be used in the upscaling method is modeled as $l=1 \mathrm{~mm}$. Isotropic linear elastic properties of the bulk material are used in voxel-based fine scale models. The mean of the Poisson's ratio and elastic modulus is set to $v=0.33$ and $E=65 \mathrm{GPa}$, respectively, with a coefficient of variation of 0.05 .

The reaction forces on the top surface of the fine scale model are calculated as the fine scale responses, denoted by $y_{F M 1,2}(j)$ and $y_{F M 2,2}(j)$ for Strut Model 1 and Strut Model 2, respectively. A linear elastic FEA is conducted when a tensile strain of $0.1 \%$ is applied at each sampling point $j=1,2, \ldots, n$, where $n$ is the total number of samples. The opposite plane is fixed in the loading direction. The number of simulations to represent the uncertainty is determined to be $n=200$ after a convergence analysis of the mean and standard deviation of the reaction force obtained from the fine scale model. The coarse scale model is generated as a beam element model with the homogenized diameter $\left(D_{h}\right)$ and elastic modulus $\left(E_{h}\right)$ predicted in the two-step upscaling process using the same boundary and loading conditions in the fine scale models as shown in Fig. 4. The two-step upscaling procedure is implemented for 200 samples while considering a second order PCE for the representation of the homogenized variables.

For the validation step at the ith scale level, the validation model is determined such that it will be different from the model used in the upscaling step in order to challenge the prediction model (i.e. the coarse scale model). For the $k$ th validation site, the experiment data is denoted by $Y_{E, i}^{(k)}$ and the coarse scale model responses are denoted by $Y_{C M, i}^{(k)}$; then the validation approach presented in Section 2.5 is implemented. At the mesoscale level of the scaffold example in Fig. 3, the reconstructed strut models obtained from the CT-scan images can be considered as the validation model to minimize the experimental cost. A tensile test simulation of these validation models are carried out under a tensile strain of $0.1 \%$ and the reaction forces are obtained as experiment data, $Y_{E, 2}^{(k)}$, at the $k$ th validation site. In the FEA of the struts models, the lateral degrees of freedoms at the top and bottom surfaces of struts are also fixed. Thus, the validation model will be different from the model used in the upscaling step to challenge the problem. The coarse scale model of this validation model is also simulated with the homogenized properties using the same boundary conditions to obtain the reaction force as the coarse scale model responses $Y_{C M, 2}^{(k)}$. Then, the proposed validation metric in Section 2.5 is utilized to accept or reject the predictions of the coarse scale model. If the model predictions are accepted based on the K-S test, this process ensures that the generated strut model can mimic the reconstructed models. Thus, the coarse scale strut model and the homogenized strut properties can be used at the next level (i.e. lattice cell level) fine scale model, i.e. $X_{F M, 1}=X_{C M, 2}$, as shown in Fig. 3.

At the macroscale level ( $i=1$ in Fig. 3 ), the upscaling method is utilized again for the lattice cell model to predict the uncertainties of the homogenized coarse scale model properties $X_{C M, 1}$. For the given example, the fine scale models of the lattice specimens are generated using the homogenized diameter and elastic modulus variables predicted at the strut level, and these models are simulated for a compression test. Before developing the fine scale lattice models, the fabricated lattice specimens are investigated. It is observed that the fabricated specimens have missing struts on the corners of the structure that occur during the removal process of the specimen from the SLM machine, as seen in Fig. 5(a). To account for their effect on the overall elastic modulus of the fabricated scaffold, the compression test simulation of the fine scale lattice model was carried out by removing the corner struts from the entire lattice structure FE model (Fig. 5(b)). The fine scale model of the entire lattice structure (Fig. 5(c)) is also simulated to investigate the differences of predictions. However, these fine scale models are still computationally very expensive even if homogenized strut properties are used. To mitigate the computational cost, a unit BCC cell model shown Fig. 5(d) is developed as the fine scale lattice model. Periodic boundary conditions (PBCs) were applied to simulate the unit cell FE model. A compression strain of $0.1 \%$ was applied in one direction to simulate the compression test simulation. Quadratic tetrahedral elements were used to mesh the fine scale lattice models. A hexagonal brick element the same size as one BCC cell is modeled with PBCs as the coarse scale model, and the corresponding elastic modulus is projected onto a second order PCE in the upscaling process.

Then, the validation process is implemented again at the top of the validation pyramid in Fig. 3 for the fabricated lattice specimens. The physical compression tests are conducted to obtain the experimental results $Y_{E, 1}^{(k)}$ at the $k$ th validation site. The corresponding FEA results $Y_{C M, 1}^{(k)}$ are obtained from the coarse scale model of the fabricated specimens with the homogenized lattice level properties. These results are compared with the experimental results at the lattice level validation step to ensure that the model predictions are acceptable based on 


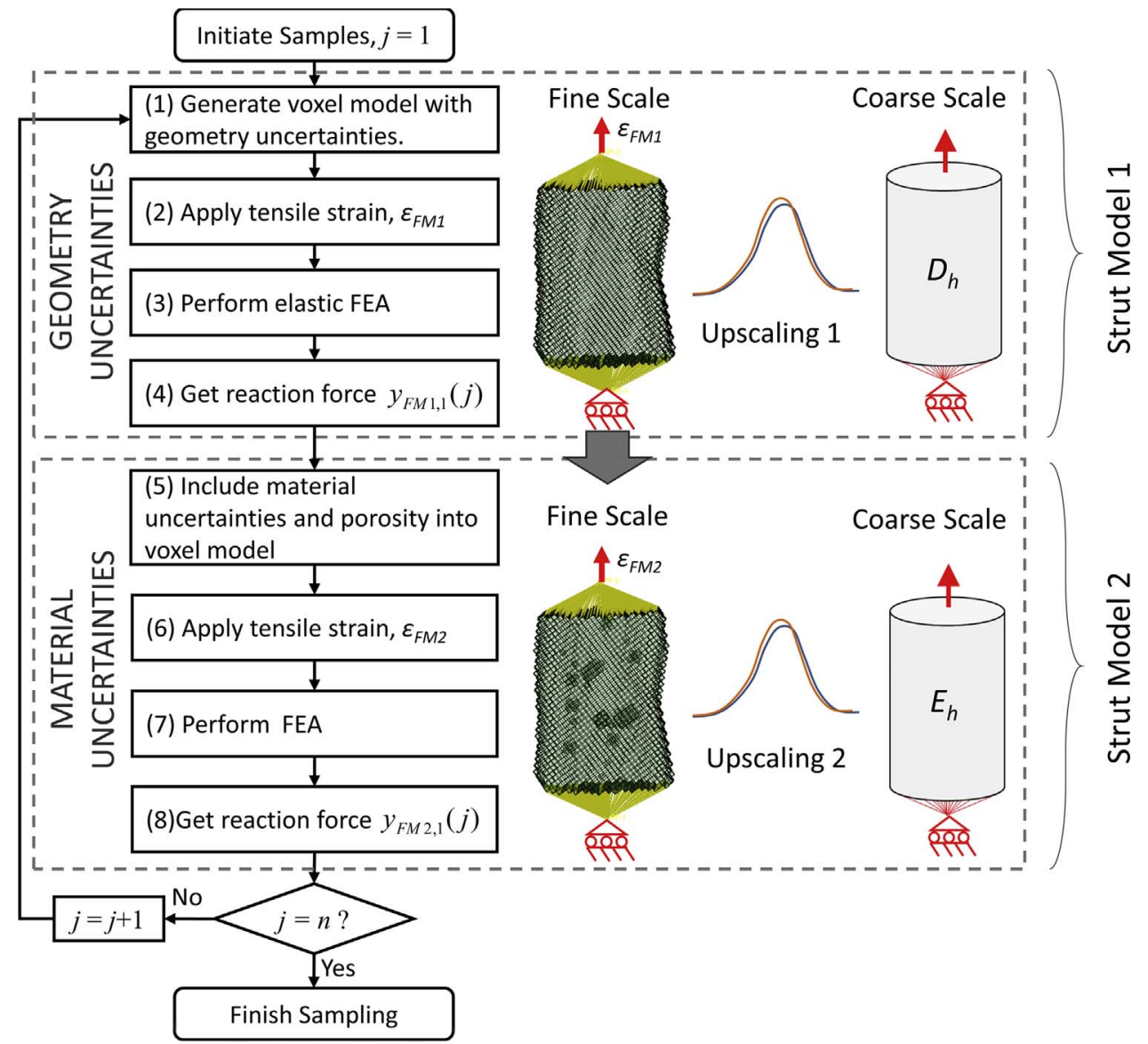

Fig. 4. Mesoscale (i.e. Strut) level upscaling process.

the proposed validation metric.

\section{Results}

\subsection{Morphological analysis for uncertainty quantification at strut level}

This section comprises the bottom of the validation pyramid shown in Fig. 3. The statistical data for the strut level input variables are collected by the measurements made on the reconstructed strut models. Then, the corresponding statistical parameters and distribution models are determined using BIC method.

\subsubsection{Analysis of strut diameter}

The struts in the BCC lattice structure have an angle of $35.3^{\circ}$ in the CAD model. To fabricate these strut members with a circular cross- section, elliptic layers sliced in the CAD model are built from bottom to top one by one in a stair shape struts as seen in Fig. 6(a). The reconstructed models of the fabricated struts are rotated to analyze the strut cross-section as seen in Fig. 6(b). It is observed that the crosssections of the fabricated struts are not fully circular, although circular cross-sections are designed in the CAD model. Rather, two distinctive diameter values are measured in the directions perpendicular to each other as seen in Fig. 6(b). The fabricated long strut diameter $D_{s, m, l o n g}$ and short strut diameter $D_{s, m, s h o r t}$ were measured for 15 reconstructed strut models to collect the statistical data of the fabricated strut diameters. 144 measurements were made from a total of 15 struts at different positions from bottom to top in its length direction. It was found from these measurements that the short diameter has a mean value of $D_{s, m, \text { short }}=0.672 \mathrm{~mm}$ and a standard deviation of $0.0193 \mathrm{~mm}$. The long diameter has a mean value of $D_{s, m, l o n g}=0.784 \mathrm{~mm}$ a standard

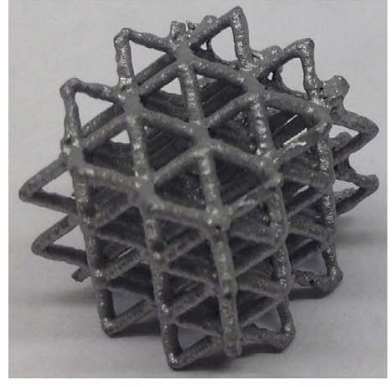

(a)

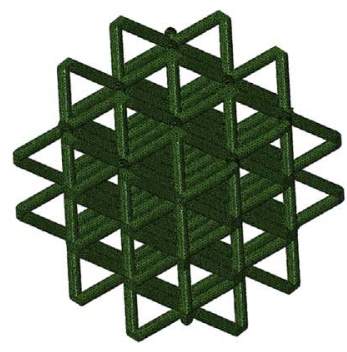

(b)

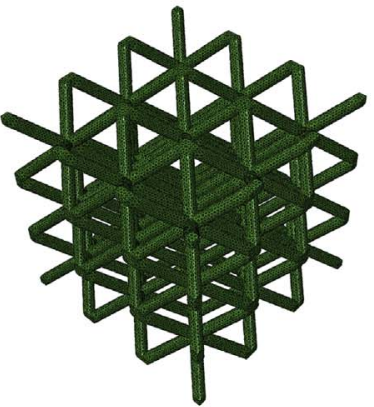

(c)

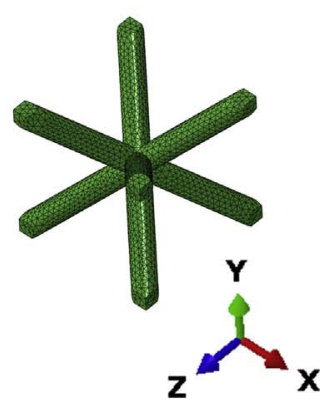

(d)

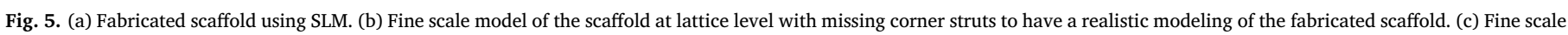
model of the entire lattice structure that is represented by the unit cell model. (d) Fine scale model of the scaffold using only one BCC unit cell to decrease the computational cost. 

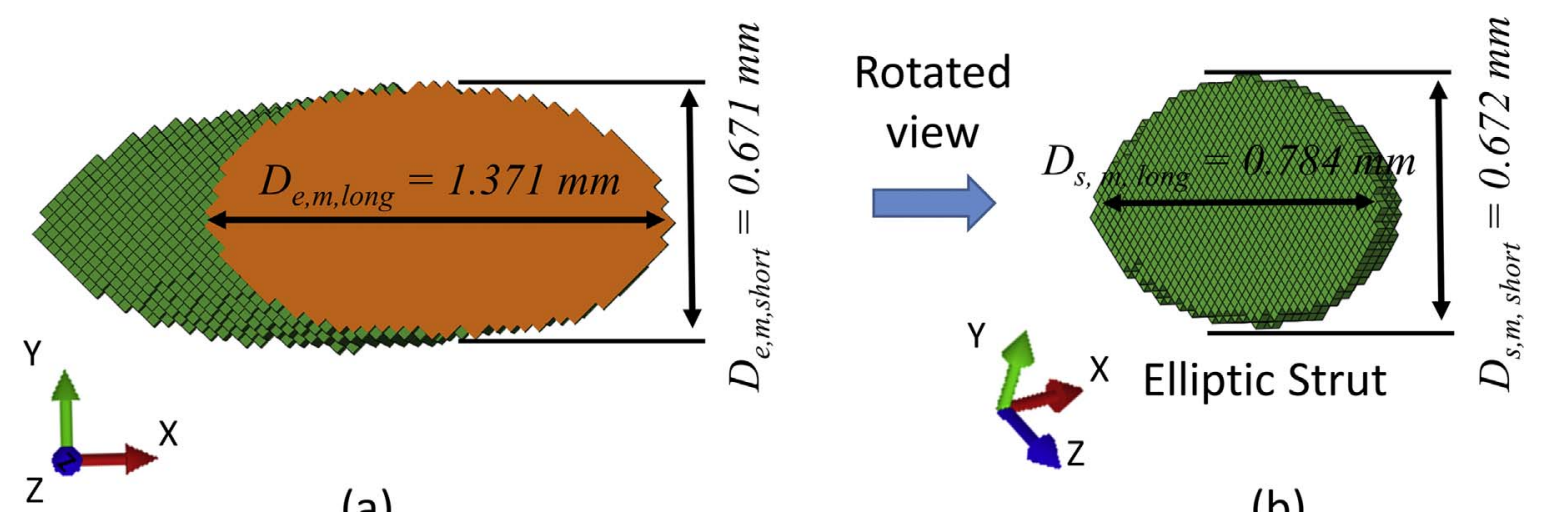

(b)

Fig. 6. Reconstructed strut model. (a) The stair-shape with an elliptic cross-section that produces the circular strut, (b) Fabricated strut cross-section obtained from the 3D reconstructed strut models.

Table 1

Distribution and statistical parameters for uncertain variables.

\begin{tabular}{llllll}
\hline Parameter & Distribution & Parameter $a$ & Parameter $b$ & Mean & $\begin{array}{c}\text { Standard } \\
\text { deviation }\end{array}$ \\
\hline $\begin{array}{c}\text { Short } \\
\text { diameter } \\
(\mathrm{mm})\end{array}$ & Lognormal & -0.398 & 0.0277 & 0.672 & 0.0193 \\
$\begin{array}{l}\text { Long } \\
\text { diameter } \\
(\mathrm{mm})\end{array}$ & Lognormal & -0.246 & 0.0704 & 0.784 & 0.0561 \\
$\begin{array}{l}\text { Strut angle }\left(^{\circ}\right) \\
\begin{array}{l}\text { Porosity } \\
\text { Pore Normal }\end{array}\end{array}$ & Gamma & 12.6 & 0.000718 & 0.00911 & 0.00252 \\
$\quad$ diameter & Lognormal & -2.924 & 0.437 & 0.0594 & 0.0283 \\
$(\mathrm{~mm})$ & & & & & \\
\hline
\end{tabular}

deviation of $0.0561 \mathrm{~mm}$. These quantified statistical parameters are listed in Table 1.

In addition to the diameter measurements made on the reconstructed models, the experimental strut diameters of the struts were measured using a digital Vernier caliper for 50 different struts from the fabricated lattice specimens. Very few measurements could be made for each strut since the length of the struts were small to distinguish the position of the caliper on the strut. Similar to the model measurements, two distinctive diameter values were observed for each strut. The scatter plot of the measurements is shown in Fig. 7(a). The mean and standard deviation of the diameters of these struts were plotted and compared with the measurements made from the models in Fig. 7(b).

The difference between the arithmetic means of the short diameters was found to be $1.8 \%$ and $1.1 \%$ for the long diameters, indicating that a close agreement has been obtained. Hence, it is concluded that the measurements made on the reconstructed 3D models of struts are able to quantify the uncertainties accurately. It was also observed that a difference exists between the target diameter (i.e. $D_{s, t}=0.5 \mathrm{~mm}$ ) and the fabricated short diameter $\left(D_{s, m, s h o r t}=0.672 \mathrm{~mm}\right)$. The difference was calculated as $\delta_{s, \text { short }}=0.172 \mathrm{~mm}$. This difference can be added to the target diameter value for the voxel model generation process of the struts in the upscaling process.

\subsubsection{Analysis of strut angle}

The models of the fabricated struts reveal that the strut angle deviation is large on the long diameter side because the build angle is determined on the side where the stair shape effect is visible. Hence, the measurements for the strut angle deviation are made in the long diameter direction. The red regions of the strut in Fig. 8(a) show the layers with partial elliptic cross-sections, while the green region has the full elliptic cross-sections. The deviation in the strut angle for each layer can be measured from the fully fabricated layers as shown in Fig. 8(b).

The dashed line in Fig. 8(b) depicts the target center coordinates when the target strut angle of $35.3^{\circ}$ is used. However, the center points obtained from the reconstructed models, shown by the red dots, has a deviation from the target center points. The deviation of the angle is

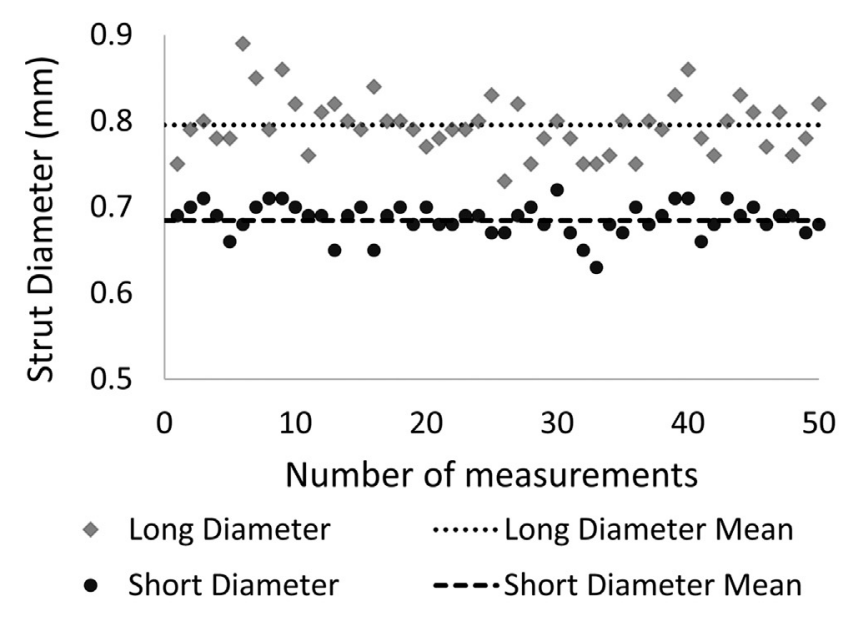

(a)

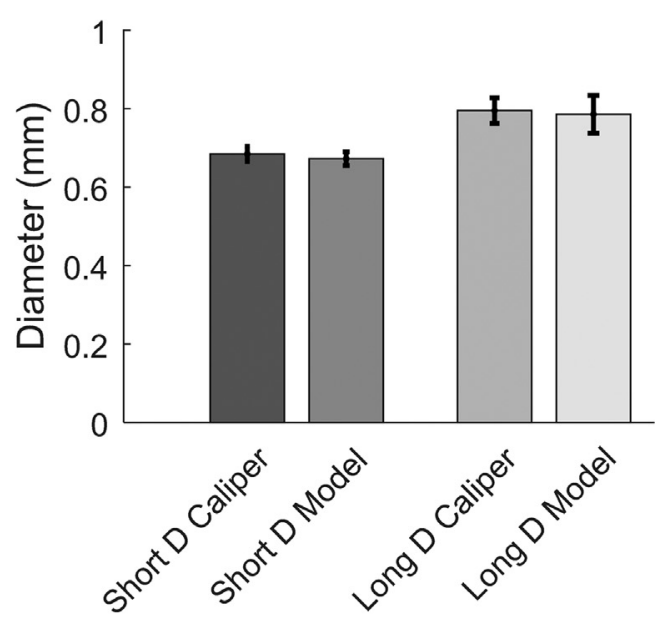

(b)

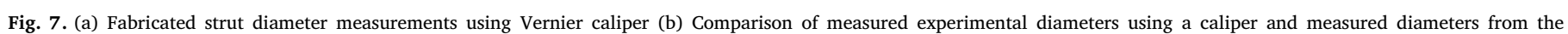
reconstructed $3 \mathrm{D}$ model. 


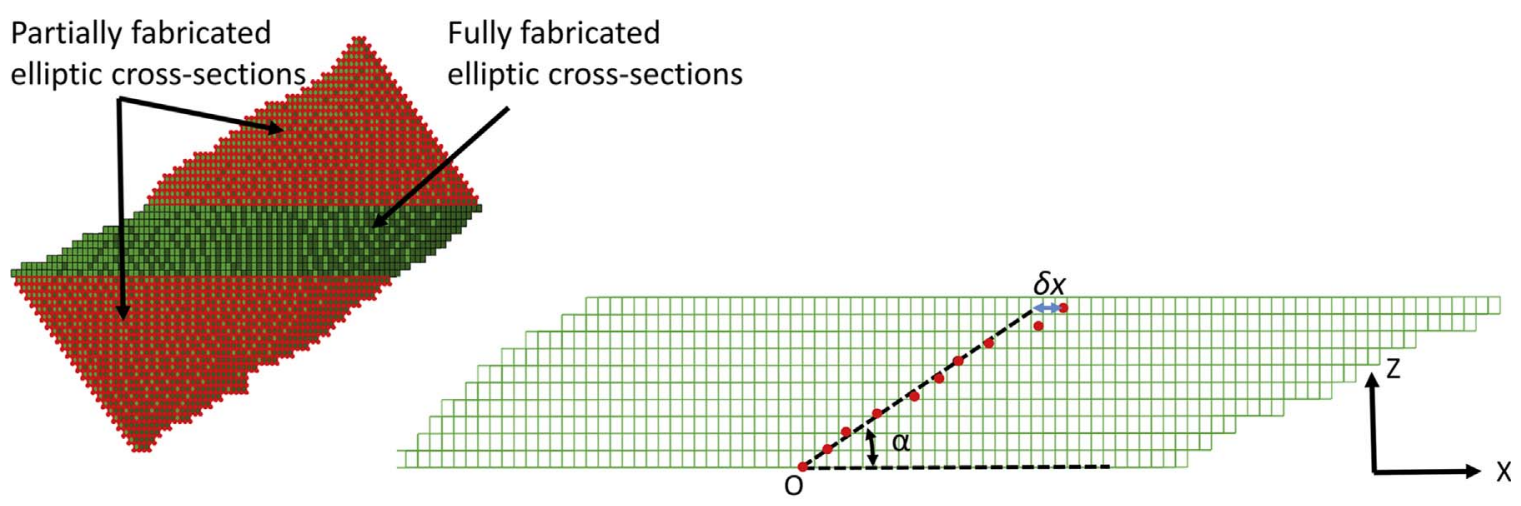

(a)

(b)

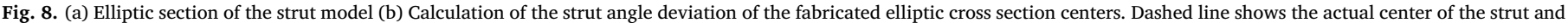
the red dots are the centers of the elliptical cross-sectional layers fabricated in SLM.

determined by measuring this deviation from the actual center points, denoted by $\delta x$ in Fig. 8(b). To find the build angle of each layer, $\alpha$, first, each layer's center value is calculated from the layer diameter. Then, the statistical data of the build angle is calculated with respect to the horizontal direction using the center coordinate values of each layer. The mean and standard deviation of the strut angle are calculated to be $34.9^{\circ}$ and $1.23^{\circ}$ as listed in Table 1.

The optimal distribution types and statistical parameters of the short diameter, long diameter, and strut angle of the fabricated struts were determined using the BIC method described in Section 2.3.1. The normal, lognormal, gamma and generalized extreme value (GEV) distributions were fitted to the measured data from the reconstructed models as shown in Fig. 9. The BIC values indicate that a lognormal distribution is the best fit to the statistical data of the short and long diameters, while a normal distribution is the best fit for the strut angle data. The corresponding statistical parameters and distribution types are listed in Table 1.

\subsubsection{Analysis of porosity and pore diameter}

3.1.3.1. Porosity distribution. The SLM process tends to produce defects in the material in the form of pores when melting layer by layer. The porosity can be determined from the reconstructed strut models as the volume fraction of the pores in the structure. It is observed that overall volume fraction of pores measured from the models is less than $1 \%$. The mean of the porosity value was found to be 0.00911 with a coefficient of variance $(\mathrm{CoV})$ value of 0.277 . BIC is implemented for the histogram of the porosity given in Fig. 10(a), and it has been found that the porosity distribution is better described with a gamma distribution with the parameters in Table 1.

3.1.3.2. Pore diameter distribution. The size of the pores can be determined by calculating the corresponding equivalent spherical diameter of the pores. For this purpose, first, the number of voxels in each pore is calculated from the reconstructed strut models. Then, the equivalent spherical diameter of each corresponding pore is calculated. The distribution of the equivalent diameter for all samples is given in Fig. 10(b). It is calculated from this statistical data that the mean pore diameter is $D_{p}=0.0594 \mathrm{~mm}$ with a standard deviation of 0.0283 , as listed in Table 1. It is seen from Fig. 10(b) that the pore size distribution is skewed to the left. This is because most of the pores in the statistical data correspond to those with only one voxel element and an equivalent sphere diameter of $0.034 \mathrm{~mm}$. In addition to normal and lognormal and gamma distributions, an exponential distribution fit is also applied, as seen in Fig. 10(b).

The BIC results indicate that a lognormal distribution has a better fit than the normal and exponential distributions. The parameters of the pore diameter distribution model are listed in Table 1. On the other hand, it is seen in Fig. 10(b) that the distributions that fit the data are truncated on the left side. Hence, in the strut model generation process, if the generated pore size is smaller than $0.034 \mathrm{~mm}$, a new value is generated from the lognormal distribution until this value falls into the truncated region of the distribution.

\subsection{Mesoscale (i.e. strut) level upscaling results}

In the first upscaling process, the fine scale model is generated by considering uncertainties only in the geometric parameters such as strut diameter and angle given in Table 1. This model is called Strut Model 1 as shown in Fig. 4. The material is assumed to be deterministic in this model with the mean values of elastic modulus and Poisson's ratio at each sampling point. In the coarse scale model, known deterministic values of elastic material properties are used while the diameter of strut, $D_{h}$, is the unknown homogenized variable. After the upscaling process, the mean and standard deviation of $D_{h}$ is found to be $0.638 \mathrm{~mm}$ and $0.00686 \mathrm{~mm}$, respectively.

The generated fine scale strut models in the first upscaling process

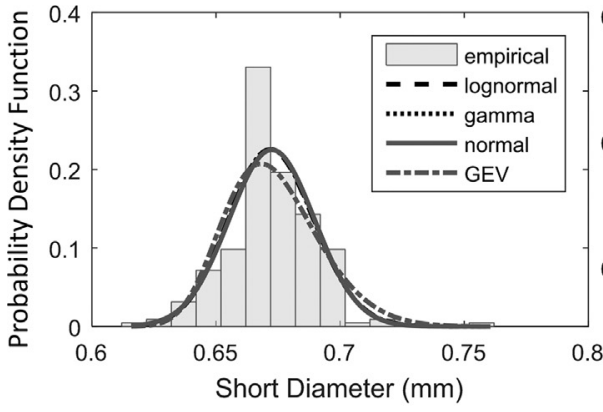

(a)

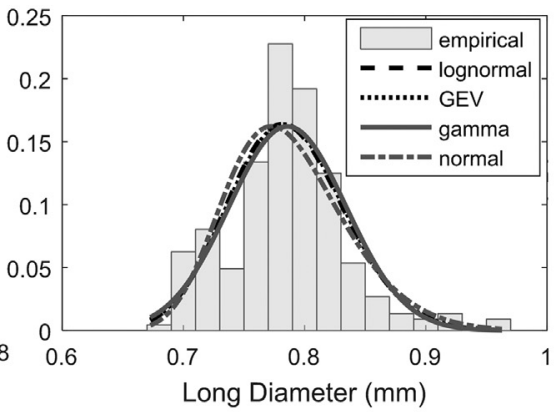

(b)

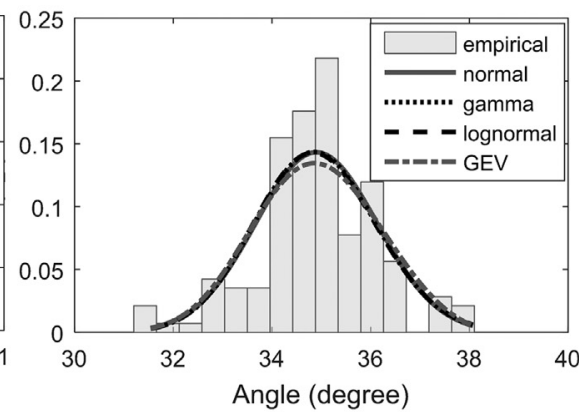

(c)

Fig. 9. (a) Distribution of the short diameter (b) Distribution of the long diameter of the strut cross section (c) Distribution of the strut angle. 


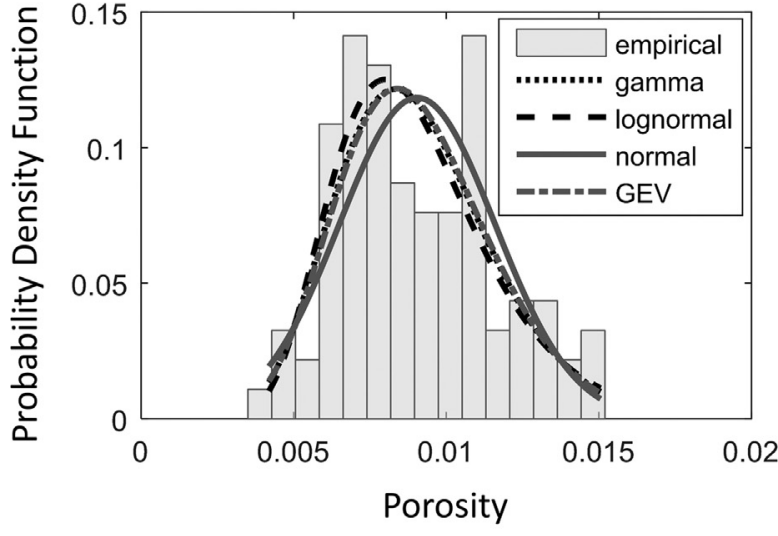

(a)

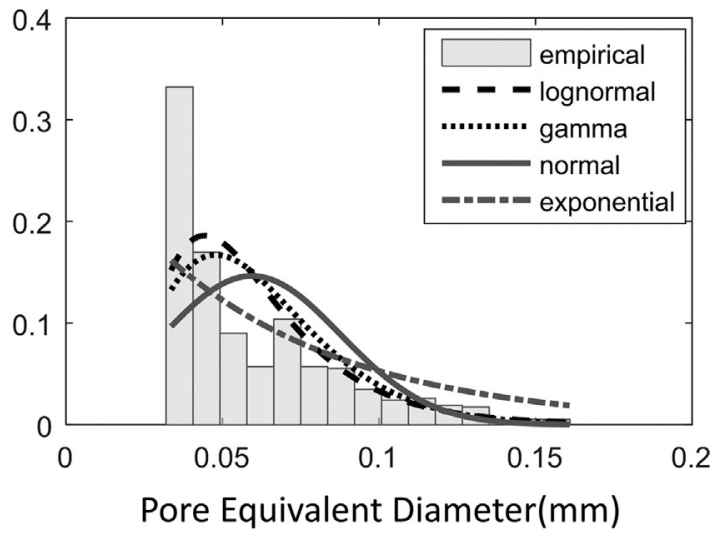

(b)

Fig. 10. (a) Porosity distribution in struts (b) Pore equivalent diameter distribution.

Table 2

Obtained statistical parameters and distribution types for the homogenized properties.

\begin{tabular}{llll}
\hline Homogenized property & Mean & Standard deviation & Distribution \\
\hline Diameter (mm) & 0.637 & 0.00686 & Normal \\
Elastic Modulus (GPa) & 63.5 & 2.44 & Normal \\
\hline
\end{tabular}

are retained for each sampling point and the uncertainties in the material properties, such as elastic modulus and porosity, are included into the fine scale strut model in the second upscaling process (i.e. Strut Model 2 in Fig. 4). It was observed from the reconstructed strut models that the pores are randomly distributed in the struts but there are no partial pores on the strut surface. Hence, the pore centers are randomly generated such that they can exist inside the strut models. In the generation of the pores at each sampling point, a porosity value is first generated from its distribution given in Table 1. The pores are generated in the strut such that the porosity value generated initially is met. The pore diameter values are also generated from its distribution in Table 1 using LHS. After this second upscaling process, the mean and standard deviation of the homogenized elastic modulus of the coarse scale model, $E_{h}$, is found to be $63.5 \mathrm{GPa}$ and $2.44 \mathrm{GPa}$, respectively. It is determined by the BIC method that the uncertainties of the homogenized variables $D_{h}$ and $E_{h}$ can be represented by using a normal distribution. The distribution types and statistical parameters of the homogenized strut level variables are listed in Table 2.

\subsection{Mesoscale (i.e. strut) level validation results}

The reconstructed strut models that are used as the validation model have a length of $1.7 \mathrm{~mm}$ and a mesh size of $27.06 \mu \mathrm{m}$, which is finer than the mesh size of $54.12 \mu \mathrm{m}$ used in the fine scale models in the upscaling method. The FEA of 20 reconstructed strut models were carried out and the total reaction force data in the loading directions were compared to the data obtained from the corresponding coarse scale strut model simulations for 200 samples for validation using the proposed validation metric described in Section 2.5. The resulting empirical CDF obtained from the $u$-pooling method agree well with the target standard uniform distribution as depicted in Fig. 11. In the K-S test, the confidence level $\alpha$ was set to 0.05 and the maximum distance between two distributions was found to be 0.182 , which produces a $p$ value of 0.469 . Since the $p$-value is greater than the $\alpha$ value, the K-S test accepts the predictions. Thus, it is concluded the predicted homogenized properties with the quantified uncertainties for the generated coarse scale strut model has good accuracy and can be used generate the lattice level fine scale models.

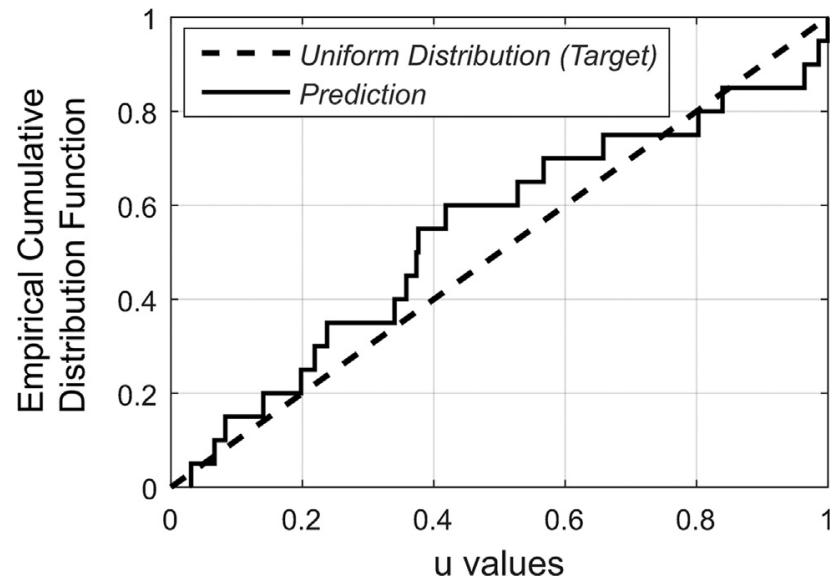

Fig. 11. Comparison of the ECDF of $u$-pooling results with SUD at strut level.

\subsection{Macroscale (i.e. lattice) level upscaling results}

A mesh convergence study was conducted to determine the optimal size of the quadratic mesh elements before using the fine scale model in the upscaling process. The deterministic values were used for the diameter and material properties in the mesh convergence study. A mesh size that was equivalent to a quarter of the diameter (i.e. $0.25 \times D$ ) was appropriate based on the mesh convergence study.

A prediction accuracy study is also needed to check if the unit cell model with the $\mathrm{PBCs}$ can produce accurate results when compared to the model of the entire lattice structure with missing struts. The unit cell model and the lattice structure model were generated using the mean values of the homogenized diameter and elastic modulus of the struts given in Table 2 . The resulting reaction forces were obtained from their deterministic FE analyses. The corresponding coarse scale model of the unit cell, which consists of only one hexahedral element with the BCC cell dimensions, was also simulated with the PBCs. The elastic modulus value of this coarse scale model $\left(E_{h}\right)$ that enables the match between the coarse scale model reaction force and fine scale model reaction force was determined under a compression strain of $0.1 \%$. Similarly, the coarse scale model of the entire lattice structure was generated by $3 \times 3 \times 3$ hexagonal elements and the corresponding coarse scale model elastic modulus was determined. The predicted elastic modulus values from the three fine scale modeling techniques shown in Fig. 5 are normalized by dividing by the bulk material elastic modulus, $E_{0}$, and compared in Fig. 12.

The results in Fig. 12 show that the lattice structure model with missing corners, which is the model of the fabricated specimens, has the smallest elastic modulus value. The relative error compared to the 


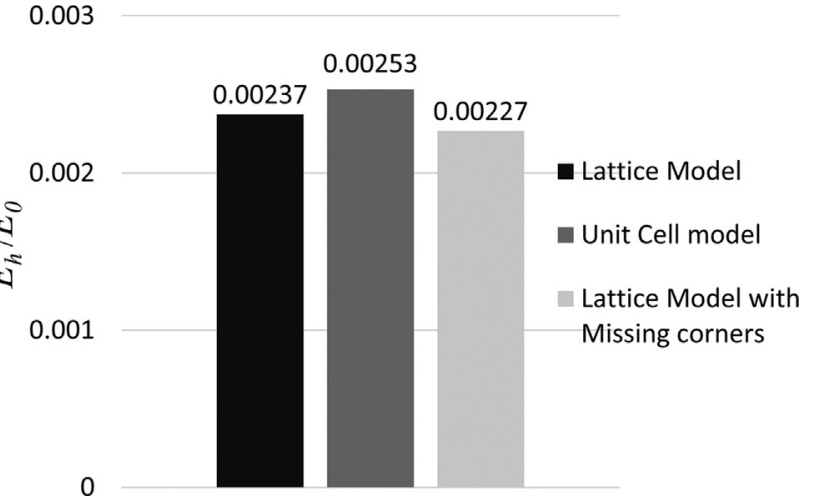

Fig. 12. Normalized elastic modulus results obtained by the three fine scale modeling approaches.

lattice model prediction was found to be $4.5 \%$. It was also found that the unit cell model with PBCs produces an elastic modulus value greater than that of the lattice structure model. The relative error between the unit cell model and the lattice structure model was calculated as $6.3 \%$. Furthermore, the relative error between the unit cell model and the lattice model with missing corner struts was $10.5 \%$. That means at the lattice level, the homogenized statistical elastic modulus data of the lattice structure, which will be used in the validation step to compare with the experiments, can be found as $89.5 \%$ of the homogenized elastic modulus data found by upscaling method with the unit cell model.

The fine scale model of the lattice structure, which is the unit BCC cell with PBCs, is simulated for 200 samples generated for the homogenized properties given in Table 2. For each sample, the diameter and elastic modulus of each strut in the unit cell were assumed to be the same but changing with the sampling point depending on the distributions of the homogenized strut diameter and elastic modulus. The total reaction force value on one face of the unit cell is obtained as the fine scale response, $Y_{F M, 1}$. The unknown elastic modulus data of the coarse scale model is obtained from the PCE predicted by the upscaling process. The corresponding mean and standard deviation of the homogenized elastic modulus of the lattice structure are calculated as $0.154 \mathrm{GPa}$ and $0.00943 \mathrm{GPa}$ when the $10.5 \%$ modeling error is considered. BIC resulted in a Gamma distribution to represent the uncertainty with the statistical parameters given in Table 3.

\subsection{Macroscale (i.e. lattice) level validation results}

For validation, the compression tests of four fabricated lattice specimens are conducted and the corresponding compression curves are shown in Fig. 13. Strains were taken as $\varepsilon_{a}=0.01$ and $\varepsilon_{b}=0.025$ from the linear region of the compression curves and experimental elastic modulus values are calculated using Eq. (1). The mean of the modulus was found to be $0.153 \mathrm{GPa}$ while its standard deviation was 0.00818 as given in Table 3. Compared to the predicted modulus mean from the lattice level upscaling method that considers the modeling error, the mean value of the predicted data has only a $1.3 \%$ difference, indicating that the predictions agree well with the experiments.

In order to evaluate the predictive capability of the coarse scale

Table 3

Statistical parameters and distribution types for the homogenized properties of BCC cell.

\begin{tabular}{llllll}
\hline $\begin{array}{l}\text { Lattice elastic } \\
\text { modulus (MPa) }\end{array}$ & Mean & $\begin{array}{l}\text { Standard } \\
\text { deviation }\end{array}$ & Distribution & Parameter a & Parameter $b$ \\
\hline $\begin{array}{l}\text { Experiment } \\
\text { Upscaling }\end{array}$ & 0.153 & 0.00818 & - & - & - \\
\hline
\end{tabular}

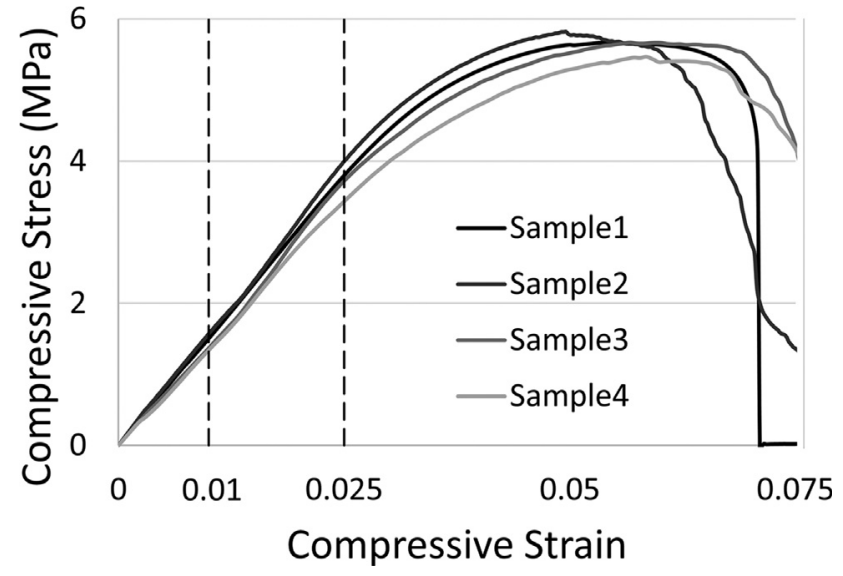

Fig. 13. Compression test curves of four lattice structure specimens.

model of the lattice structure, the predicted responses from the compression simulation of the lattice structure must be compared to the experimental results rather than evaluating the predicted homogenized elastic modulus. For this purpose, a coarse scale model of the entire lattice structure specimen was generated, as illustrated at the top level of the validation pyramid in Fig. 3. 200 samples were generated for the homogenized elastic modulus, and compression simulations were carried out for the coarse scale lattice model. A compression strain of $0.1 \%$ was applied in the simulations and the reaction force $\left(Y_{C M, 1}^{(1)}\right)$ was obtained from 200 simulations. The BIC method was used to predict the distribution model of the reaction force data. The experimental reaction force values for $0.1 \%$ strain were obtained from the compression curves of experiments. There exist $n_{e}=4$ experiments performed for one validation site (i.e. $n_{V S}=1$ ), which gives $n_{T}=4$ experimental results in total. The corresponding empirical CDF obtained by the $u$-pooling method is compared with the target SUD in Fig. 14(a). The K-S test was implemented for the $u$-pooling results obtained from three different modeling approaches, i.e. the entire lattice model, unit cell model, and unit cell model by accounting for the modeling error. The results of the K-S test are listed in Table 4 for each modeling approach.

The results in Fig. 14(a) show that the ECDF predictions made by the unit cell model, shown by the dash-dot line, largely deviates from the target distribution if the modeling error is not considered. On the other hand, the CDFs of the full lattice model predictions and unit cell model predictions with modeling error tend to follow the target distribution, as shown by a dotted line and solid line, respectively. According to the K-S test results in Table 4, the CDFs of all models are accepted as the corresponding $p$-values are smaller than the $\alpha$ value, which is set to 0.05 .

In addition, three validation sites are considered (i.e. $n_{V S}=3$ ) to increase the number of experimental observations. The coarse scale model of the scaffold specimen was simulated for three compression strain values, which are in the elastic region of the compressive curves obtained from the experiments: (1) A compression strain of $\varepsilon_{C M, 1}^{(1)}=0.001$ was applied and the reaction force data. $\left(Y_{C M, 1}^{(1)}\right)$ was obtained from 200 simulations. (2) A compression strain of $\varepsilon_{C M, 1}^{(2)}=0.01$ was acquired to obtain the reaction force data $\left(Y_{C M, 1}^{(2)}\right)$ as the second validation site. (3) A compression strain of $\varepsilon_{C M, 1}^{(3)}=0.025$ was applied to compute the reaction force data $\left(Y_{C M, 1}^{(3)}\right)$ as the third validation site. These three strain values are considered because the first was used in upscaling to predict the homogenized elastic modulus while the second and third values were used to calculate the experimental elastic modulus value with Eq. (1). There exist four experimental reaction force values, $n_{e}=4$, for each validation site, which gives $n_{T}=12$ experimental results in total. Then, the $u$-pooling method was used to find the ECDFs of all validation sites for three modeling approaches. Those results are compared with the target SUD in Fig. 14(b). Then, The 


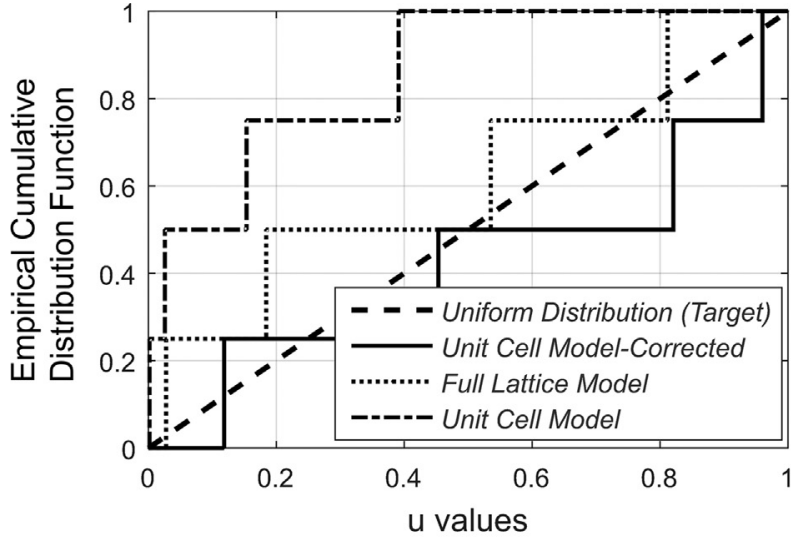

(a)

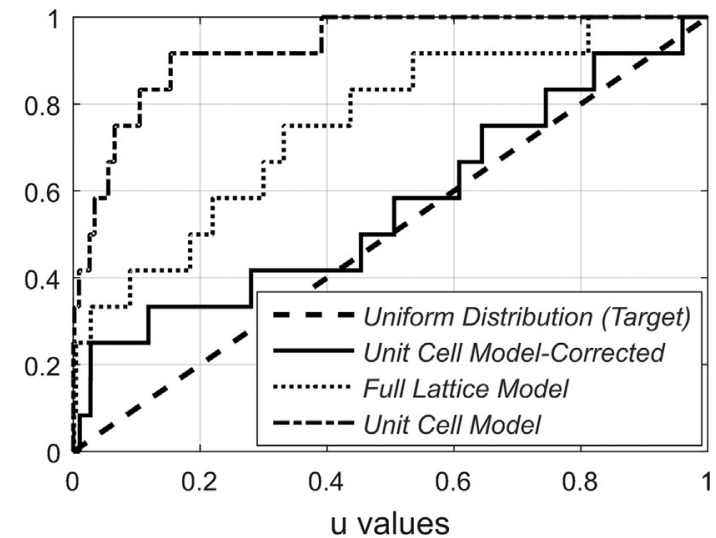

(b)

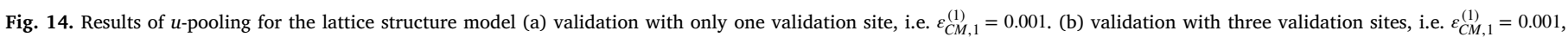
$\varepsilon_{C M, 1}^{(2)}=0.01$ and $\varepsilon_{C M, 1}^{(3)}=0.025$.

Table 4

K-S test results for one validation sites, i.e. $\varepsilon_{C M, 1}^{(1)}=0.001$

\begin{tabular}{llll}
\hline Model & K-S Statistic & p-value & Decision \\
\hline Unit cell & 0.609 & 0.061 & Accept \\
Full lattice structure & 0.316 & 0.721 & Accept \\
Unit cell and modeling error & 0.321 & 0.704 & Accept \\
\hline
\end{tabular}

Table 5

K-S test results for three validation sites, i.e. $\varepsilon_{C M, 1}^{(1)}=0.001, \varepsilon_{C M, 1}^{(2)}=0.01$, and $\varepsilon_{C M, 1}^{(3)}=0.025$.

\begin{tabular}{llll}
\hline Model & K-S Statistic & p-value & Decision \\
\hline Unit cell & 0.763 & 0.000826 & Reject \\
Full lattice structure & 0.418 & 0.0204 & Reject \\
Unit cell and modeling error & 0.223 & 0.521 & Accept
\end{tabular}

K-S test was implemented and the decision results three validation sites are listed in Table 5.

As seen in Fig. 14(b), similar to the one validation site results, the ECDF of the unit cell model predictions, shown by a dot-dash line, also deviates largely from the target distribution for three validation sites. Unlike the case of one validation site, on the other hand, the ECDF of the full lattice model, shown by dotted line, also deviates from the SUD for three validation sites. Only the ECDF of the unit cell model that accounts for the modeling error, shown by the solid line, has the following trend with the SUD. It is also seen from the results in Table 5 that the CDFs of the full lattice structure model predictions and unit cell model predictions are rejected, as the calculated $p$-value is smaller than $\alpha=0.05$ value. On the other hand, in Table 5, the K-S statistic value is the smallest for the unit cell model with modeling error (i.e. 0.223 ) and K-S test only accepted the predictions made by the unit cell model with the modeling error considered.

\section{Discussions}

Local geometric variations and uncertainties of the struts have considerable influence on the effective material properties of the scaffolds designed by cellular lattice structures. The realistic design of scaffolds is therefore only possible when accurate material data is available. On the other hand, gathering accurate material data that is used in the design of scaffolds can be costly for all different strut sizes. To address this issue, this paper presents a methodology that can quantify uncertainties at multiple scale levels to improve the predictive capability of the computational models and capture the real mechanical behavior of lattice scaffolds fabricated by AM processes. As a non- destructive experimental technique, the micro-CT scan was successfully applied to obtain cross-sectional images of lattice structures fabricated by the SLM process. The reconstructed 3D dataset obtained from the preprocessed 2D images was applied to characterize the uncertainties on the geometric variations on the strut surface and the pore structure in strut material. The uncertainties on the struts were identified based on the individual strut models reconstructed from the scaffold specimens instead of the additionally fabricated strut specimens, greatly decreasing the experimental cost and effort to characterize the strut level properties. The corresponding uncertainties on the strut diameter, angle, and porosity are successfully quantified with parametric distribution models using the BIC method and used for FE model development of struts to achieve inherent relationships of pores and strut geometry. Thus, it became possible to generate and simulate a realistic 3D strut model of the fabricated structure using the statistical sampling method to minimize the experimental cost.

A multi-level validation approach that incorporates the $u$-pooling method with the K-S test is implemented, along with a stochastic upscaling process for the characterization of lattice structure mechanical properties with quantified uncertainties. The applied procedure has successfully identified the uncertainties on the homogenized diameter and elastic modulus of the struts at the mesoscale level while ensuring the accurate matching of the responses between the fine and coarse scale models. At the macroscale level, on the other hand, because there were only for lattice scaffold experiments, a large acceptance margin is calculated by K-S test, which resulted in accepting the predictions of all three models as shown in Table 4. In addition, the full lattice structure and unit cell models with modeling error have very close K-S statistic values ( 0.316 and 0.321 , respectively), which are smaller than the value obtained for the unit cell model without the modeling error. This means that the former two models provide better predictions. However, since the K-S test statistic values are very close for these two, a comparison between them is not possible for one validation site. Thus, these observations suggest that using one validation site where only four experimental results exist may not be effective in prediction assessment. The number of experiments must be increased to have more reliable decisions; however, this will increase the experimental cost. A better method is to use multiple validation sites from the same experiments to minimize the experimental cost as in the definition of the $u$-pooling method (Ferson et al., 2008), i.e. to obtain experimental results for different strain values. When three validation sites are considered to use this advantage, it is seen from the K-S test results in Table 5 that the accepted unit cell model with the modeling error can predict the responses accurately in the elastic region of the compression curve of the lattice specimen, while the other models fail to make accurate predictions. Thus, the results suggest that the accepted unit 
cell model with modeling error can provide accurate predictions for the scaffold specimen.

These results clearly show the advantage of the proposed validation approach that utilizes multiple validation sites and the K-S test: Having multiple validation sites is very effective in minimizing the experimental cost and distinguishing model predictions because there were twelve experimental results in this example that narrowed down the acceptance margin of the K-S test, and also enabled better representation of the CDFs even though only a few physical experiments are conducted. Thus, these results prove that the proposed methodology that utilizes the uncertainty quantification approach using the 3D strut models reconstructed from the CT scan images, the stochastic upscaling method, and the proposed validation approach used at multiple scale levels provides an accurate prediction of the mechanical behavior of lattice scaffolds fabricated by AM with minimal experimental cost.

\section{Conclusion}

A methodology is presented in this study to quantify the uncertainties introduced by the AM-processes on the fabricated strut members of the lattice scaffolds and to validate the predictions of the computational models used to predict final scaffold performance under these uncertainties. The results of the example lattice scaffold application indicate that the applied procedure has successfully propagated the uncertainties at both strut level and lattice structure level while ensuring accurate matching of responses between fine and coarse scale models. The simulated lattice structures with homogenized properties obtained from the upscaling process with the quantified uncertainties gave a good estimate with the test data obtained for lattice scaffold specimens when the modeling error is considered in computations. These observations show that the systematic validation approach that accounts for uncertainties with minimal experimental cost is critical for multi-level modeling of lattice structures. Although the presented metal scaffold example is fabricated from the SLM process, the proposed approach can be applicable to most AM processes and is not limited to specific materials. Hence, the presented uncertainty quantification and multilevel validation procedure has significant potential to effectively certify the lattice scaffolds fabricated by AM while minimizing the experimental cost, thus enabling the efficient consideration of uncertainties in the design of the AM-fabricated parts for critical medical applications.

\section{Acknowledgement}

This work was partially supported by the National Science Foundation under Grant Number CMMI-1538744.

\section{References}

Arabnejad, K., S., Pasini, D., 2012. Multiscale design and multiobjective optimization of orthopedic hip implants with functionally graded cellular material. J. Biomech. Eng. 134, 31004. http://dx.doi.org/10.1115/1.4006115.

Arabnejad, S., Burnett Johnston, R., Pura, J.A., Singh, B., Tanzer, M., Pasini, D., 2016. High-strength porous biomaterials for bone replacement: a strategy to assess the interplay between cell morphology, mechanical properties, bone ingrowth and manufacturing constraints. Acta Biomater. 30, 345-356. http://dx.doi.org/10.1016/ j.actbio.2015.10.048.

Babuška, I., Nobile, F., Tempone, R., 2007. Reliability of computational science. Numer. Methods Partial Differ. Equ. 23, 753-784. http://dx.doi.org/10.1002/num.20263.

Beck, J.L., Yuen, K., 2004. Bayesian probabilistic approach. J. Eng. Mech. 130, 192-203. http://dx.doi.org/10.1061/(ASCE)0733-9399(2004)130:2(192).

Cahill, S., Lohfeld, S., McHugh, P.E., 2009. Finite element predictions compared to experimental results for the effective modulus of bone tissue engineering scaffolds fabricated by selective laser sintering. J. Mater. Sci. Mater. Med. 20, 1255-1262. http://dx.doi.org/10.1007/s10856-009-3693-5.

Campoli, G., Borleffs, M.S., Amin Yavari, S., Wauthle, R., Weinans, H., Zadpoor, A.A., 2013. Mechanical properties of open-cell metallic biomaterials manufactured using additive manufacturing. Mater. Des. 49, 957-965. http://dx.doi.org/10.1016/j. matdes. 2013.01.071.

Cansizoglu, O., Harrysson, O., Cormier, D., West, H., Mahale, T., 2008. Properties of Ti$6 \mathrm{Al}-4 \mathrm{~V}$ non-stochastic lattice structures fabricated via electron beam melting. Mater. Sci. Eng. A 492, 468-474. http://dx.doi.org/10.1016/j.msea.2008.04.002.
Challis, V.J., Roberts, A.P., Grotowski, J.F., Zhang, L.C., Sercombe, T.B., 2010. Prototypes for bone implant scaffolds designed via topology optimization and manufactured by solid freeform fabrication. Adv. Eng. Mater. 12, 1106-1110. http://dx.doi.org/10. 1002/adem.201000154.

Choi, S.-K., Grandhi, R.V., Canfield, R.A., 2006. Reliability-based Structural Design. Springer Science \& Business Media, London.

Deshpande, V.S., Fleck, N.A., Ashby, M.F., 2001. Effective properties of the octet-truss lattice material. J. Mech. Phys. Solids 49, 1747-1769. http://dx.doi.org/10.1016/ S0022-5096(01)00010-2.

Doyle, H., Lohfeld, S., Dürselen, L., McHugh, P., 2015. Computational modelling of ovine critical-sized tibial defects with implanted scaffolds and prediction of the safety of fixator removal. J. Mech. Behav. Biomed. Mater. 44, 133-146. http://dx.doi.org/10. 1016/j.jmbbm.2015.01.008.

Doyle, H., Lohfeld, S., McHugh, P., 2014. Predicting the elastic properties of selective laser sintered PCL/ $\beta$-TCP bone scaffold materials using computational modelling. Ann. Biomed. Eng. 42, 661-677. http://dx.doi.org/10.1007/s10439-013-0913-4.

Eshraghi, S., Das, S., 2012. Micromechanical finite-element modeling and experimental characterization of the compressive mechanical properties of polycaprolactone hydroxyapatite composite scaffolds prepared by selective laser sintering for bone tissue engineering. Acta Biomater. 8, 3138-3143. http://dx.doi.org/10.1016/j. actbio.2012.04.022.

Ferson, S., Oberkampf, W.L., Ginzburg, L., 2008. Model validation and predictive capability for the thermal challenge problem. Comput. Methods Appl. Mech. Eng. 197, 2408-2430. http://dx.doi.org/10.1016/j.cma.2007.07.030.

Giannitelli, S.M., Accoto, D., Trombetta, M., Rainer, A., 2014. Current trends in the design of scaffolds for computer-aided tissue engineering. Acta Biomater. 10, 580-594. http://dx.doi.org/10.1016/j.actbio.2013.10.024.

Gorguluarslan, R.M., Choi, S., 2014. A Simulation-Based Upscaling Technique for Multiscale Modeling of Engineering Systems Under Uncertainty. 12, pp. 549-566. 〈http://dx.doi.org/10.1615/IntJMultCompEng.2014011519〉.

Gorguluarslan, R.M., Gandhi, U.N., Mandapati, R., Choi, S.K., 2016. Design and fabrication of periodic lattice-based cellular structures. Comput. Aided. Des. Appl. 13, 50-62. http://dx.doi.org/10.1080/16864360.2015.1059194.

Gorguluarslan, R.M., Park, S.-I., Rosen, D.W., Choi, S.-K., 2015. A multilevel upscaling method for material characterization of additively manufactured part under uncertainties. J. Mech. Des. 137, 111408. http://dx.doi.org/10.1115/1.4031012.

Hammetter, C.I., Rinaldi, R.G., Zok, F.W., 2013. Pyramidal lattice structures for high strength and energy absorption. J. Appl. Mech. 80, 41015. http://dx.doi.org/10. $1115 / 1.4007865$.

Harrysson, O.L.A., Cansizoglu, O., Marcellin-Little, D.J., Cormier, D.R., West, H.A., 2008. Direct metal fabrication of titanium implants with tailored materials and mechanical properties using electron beam melting technology. Mater. Sci. Eng. C 28, 366-373. http://dx.doi.org/10.1016/j.msec.2007.04.022.

Heinl, P., Müller, L., Körner, C., Singer, R.F., Müller, F.A., 2008. Cellular Ti-6Al-4V structures with interconnected macro porosity for bone implants fabricated by selective electron beam melting. Acta Biomater. 4, 1536-1544. http://dx.doi.org/10. 1016/j.actbio.2008.03.013.

Hollister, S.J., 2009. Scaffold design and manufacturing: from concept to clinic. Adv. Mater. 21, 3330-3342. http://dx.doi.org/10.1002/adma.200802977.

Karamooz Ravari, M.R., Kadkhodaei, M., Badrossamay, M., Rezaei, R., 2014. Numerical investigation on mechanical properties of cellular lattice structures fabricated by fused deposition modeling. Int. J. Mech. Sci. 88, 154-161. http://dx.doi.org/10. 1016/j.ijmecsci.2014.08.009.

Kowalczyk, P., 2001. Design optimization of cementless femoral hip prostheses using finite element analysis. J. Biomech. Eng. 123, 396-402. http://dx.doi.org/10.1115/ 1.1392311.

Li, J.P., de Wijn, J.R., Van Blitterswijk, C.A., de Groot, K., 2006. Porous Ti 6 Al 4 V scaffold directly fabricating by rapid prototyping: preparation and in vitro experiment. Biomaterials 27, 1223-1235. http://dx.doi.org/10.1016/j.biomaterials. 2005.08.033.

Marsaglia, G., Tsang, W.W., Wang, J., 2003. Evaluating Kolmogorov's distribution. J. Stat. Softw. 8, 1-4. http://dx.doi.org/10.18637/jss.v008.i18.

Mckay, M.D., Beckman, R.J., Conover, W. J., 2000. Three Methods for A Comparison Selecting Variables in the Analysis of Values of Input a Computer From Code Output. 42, pp. 55-61. 〈http://dx.doi.org/10.1080/00401706.2000.10485979〉.

Mullen, L., Stamp, R.C., Fox, P., Jones, E., Ngo, C., Sutcliffe, C.J., 2010. Selective laser melting: a unit cell approach for the manufacture of porous, titanium, bone in-growth constructs, suitable for orthopedic applications. II Randomized structures. J. Biomed. Mater. Res. - Part B Appl. Biomater. 92, 178-188. http://dx.doi.org/10.1002/jbm.b. 31504.

Myung, I.J., 2003. Tutorial on maximum likelihood estimation. J. Math. Psychol. 47, 90-100. http://dx.doi.org/10.1016/S0022-2496(02)00028-7.

Najm, H., 2009. Uncertainty quantification and polynomial chaos techniques in computational fluid dynamics. Annu. Rev. Fluid Mech. 41, 35-52. http://dx.doi.org/ 10.1146/annurev.fluid.010908.165248.

Oberkampf, W.L., Roy, C.J., 2010. Verification and Validation in Scientific Computing. Cambridge University Press, Cambridge.

Otsu, N., 1979. A threshold selection method from gray-level histograms. IEEE Trans. Syst. Man. Cybern. 9, 62-66. http://dx.doi.org/10.1109/TSMC.1979.4310076.

Park, S.I., Rosen, D.W., Choi, S. kyum, Duty, C.E., 2014. Effective mechanical properties of lattice material fabricated by material extrusion additive manufacturing. Addit Manuf. 1, 12-23. http://dx.doi.org/10.1016/j.addma.2014.07.002.

Parthasarathy, J., Starly, B., Raman, S., Christensen, A., 2010. Mechanical evaluation of porous titanium (Ti6Al4V) structures with electron beam melting (EBM). J. Mech. Behav. Biomed. Mater. 3, 249-259. http://dx.doi.org/10.1016/j.jmbbm.2009.10. 006 
Schwarz, G., 1978. Estimating the dimension of a model. Ann. Stat. 6, 461-464. http:// dx.doi.org/10.1214/aos/1176344136.

Senatov, F.S., Niaza, K.V., Zadorozhnyy, M.Y., Maksimkin, A.V., Kaloshkin, S.D., Estrin, Y.Z., 2016. Mechanical properties and shape memory effect of 3D-printed PLA-based porous scaffolds. J. Mech. Behav. Biomed. Mater. 57, 139-148. http://dx.doi.org/10. 1016/j.jmbbm.2015.11.036.

Shell, M.S., 2008. The relative entropy is fundamental to multiscale and inverse thermodynamic problems. J. Chem. Phys. 129, 1-8. http://dx.doi.org/10.1063/1.
2992060.

Wieding, J., Wolf, A., Bader, R., 2014. Numerical optimization of open-porous bone scaffold structures to match the elastic properties of human cortical bone. J. Mech. Behav. Biomed. Mater. 37, 56-68, http://dx.doi.org/10.1016/j.jmbbm.2014.05.002. Zein, I., Hutmacher, D.W., Tan, K.C., Teoh, S.H., 2002. Fused deposition modeling of novel scaffold architectures for tissue engineering applications. Biomaterials 23 , 1169-1185. http://dx.doi.org/10.1016/S0142-9612(01)00232-0. 